\title{
Metastasis-associated protein is a predictive biomarker for metastasis and recurrence in gastric cancer
}

\author{
YOSHINAGA OKUGAWA $^{1,2}$, YASUHIKO MOHRI ${ }^{1}$, KOJI TANAKA $^{1}$, MIKIO KAWAMURA $^{1}$, SUSUMU SAIGUSA $^{1}$, \\ YUJI TOIYAMA $^{1}$, MASAKI OHI $^{3}$, YASUHIRO INOUE ${ }^{1}$, CHIKAO MIKI $^{2}$ and MASATO KUSUNOKI ${ }^{1}$ \\ ${ }^{1}$ Department of Gastrointestinal and Pediatric Surgery, Division of Reparative Medicine, Institute of Life Sciences, \\ Mie University Graduate School of Medicine, Mie 514-8507; ${ }^{2}$ Department of Surgery and Medical Oncology, \\ Iga Municipal Ueno General Citizen's Hospital, Mie 518-0823; ${ }^{3}$ Department of Innovative Surgery, Division of Reparative \\ Medicine, Institute of Life Sciences, Mie University Graduate School of Medicine, Tsu, Mie 514-8507, Japan
}

Received February 26, 2016; Accepted March 26, 2016

DOI: 10.3892/or.2016.5054

\begin{abstract}
The metastasis-associated (MTA) gene family is a critical component of the nucleosome remodeling and histone deacetylase complex, and plays an important role in metastatic processes. We systematically evaluated dysregulation of the MTA family to clarify their clinical significance in gastric cancer (GC). One hundred and forty-five patients who underwent surgery for GC were evaluated. We analyzed the expression levels of the MTA family (MTA1, 2 and 3) by qPCR in GC tissue, and the MTA1 protein expression in primary cancer and matched normal mucosa (NM) was measured using immunohistochemical analysis. The expression of all the MTA family members was significantly increased in a stage-dependent manner, and elevated expression of all of the MTA family members was correlated with metastatic factors and prognosis in GC patients. Multivariate analysis revealed that MTA1 overexpression was an independent risk factor for survival. Especially, elevated expression of MTA1 was significantly correlated with recurrence, and was an independent risk factor for lymph node metastasis. Immunohistochemical analysis demonstrated that MTA1 was predominantly expressed in the nuclei of primary GC cells but was not expressed in $\mathrm{NM}$ and in the cancer stroma. In conclusion, quantification of MTA expression may support the accurate diagnosis of disease staging and may help predict clinical outcomes.
\end{abstract}

Correspondence to: Dr Yoshinaga Okugawa, Department of Gastrointestinal and Pediatric Surgery, Division of Reparative Medicine, Institute of Life Sciences, Mie University Graduate School of Medicine, 2-174 Edobashi, Tsu, Mie 514-8507, Japan

E-mail: yosinaga@clin.medic.mie-u.ac.jp

Key words: metastasis-associated gene family, gastric cancer, lymph node metastasis, recurrence, prognosis

\section{Introduction}

Gastric cancer (GC) is the fourth most common cancer and the second leading cause of cancer-related deaths worldwide (1). Although significant progress has been made in the diagnosis and treatment of GC patients, one-third of these patients have already reached late-stage GC with distant metastasis at the time of diagnosis, and prognosis remains poor $(1,2)$. Identification of critical factors driving the progression of metastasis and new prognostic biomarkers are urgently required to improve both early detection of recurrence and prognosis of patients with advanced GC. Furthermore, in association with recent advances in surgical techniques including endoscopy and laparoscopy procedures, identification of GC patients with lymph node metastasis is also needed to relieve surgical stress and improve the quality of life of early-stage GC patients. Therefore, identification of novel biomarkers for detecting metastasis is a major focus of current investigations.

Metastasis from the primary site is a characteristic event in tumor development and is established through multiple steps, including migration of tumor cells through the stroma, invasion of vessels into the circulation, adherence to the microvascular endothelium, extravasation, and proliferation in the target organ (3-6). Although accumulating evidence over the past decades has identified hundreds of molecules that are intimately involved in metastasis, the metastasis-associated (MTA) tumor gene family has attracted more attention because it is one of the most important players in the multistep invasion-metastasis cascade (7-9). The MTA protein family mainly has three well-known members, MTA1, 2 and 3, and dysregulation of the MTA family is intimately involved in cancer biology via regulation of downstream oncogene or onco-suppressor genes (10).

A previous study from our group has shown that several MTAs are differentially expressed in advanced GC, and can be used as biomarkers for prognosis and metastasis prediction in GC patients (11-15). Although a growing number of studies have demonstrated the function of the MTA family in other types of cancer, no reports have shown any clinical significance between MTA family expression and GC progression. In this study, we systematically investigated the 
comprehensive expression profile of the MTA family using a large cohort of specimens to clarify their clinical significance as prognostic biomarkers and predictive biomarkers for lymph node metastasis and recurrence in GC patients.

\section{Materials and methods}

Patients and sample collection. A total of 145 patients (116 men and 29 women) were included in our study according to the availability of cancer tissue samples with complete clinical data and the quality of isolated RNA for real-time PCR, and were consecutive patients who underwent surgery for GC from 2000 to 2009 at Mie University Hospital, Japan. The mean age was 67 years (range, 18-90 years). No patient received chemotherapy or radiotherapy prior to surgery and no perioperative mortalities were observed. The diagnosis of GC was confirmed for all 145 patients based on clinicopathological findings. All patients were classified according to the Japanese Classification of Gastric Carcinoma (16) such that 21 patients had stage I disease, 39 had stage II, 42 had stage III, and 43 were stage IV. Ninety-nine patients received curative resection while 46 underwent non-curative resection. Distal or total gastrectomy with D2 lymphadenectomy was performed in patients who underwent curative resection. Patients with liver, peritoneal, or distant metastasis underwent palliative gastrectomy with D1 lymphadenectomy. The mean follow-up was 26 months (range, 1-79 months). During the study period, 64 patients died from cancer-related causes.

All tissue specimens were preserved immediately after surgical resection in RNAlater (Qiagen, Chatsworth, CA, USA) and stored at $-80^{\circ} \mathrm{C}$ until RNA extraction. Written informed consent was obtained from each patient, and the study was approved by the Institutional Review Boards of all the involved institutions.

Total RNA extraction and cDNA synthesis. RNAlater-preserved surgical specimens were homogenized with a Mixer Mill MM 300 homogenizer (Qiagen). Total RNA from tissues was isolated using RNeasy Mini kits (Qiagen) according to the manufacturer's instructions. cDNA was synthesized from $5.0 \mu \mathrm{g}$ of total RNA with random hexamer primers and SuperScript III reverse transcriptase (Invitrogen Life Technologies ${ }^{\mathrm{TM}}$, Carlsbad, CA, USA).

Real-time quantitative RT-PCR. Quantitative reverse transcriptase PCR (qRT-PCR) analysis was performed using the StepOne Real-Time PCR system (Applied Biosystems, Foster City, CA, USA). The MTA family (MTA1, 2 and 3) and glyceraldehyde 3-phosphate dehydrogenase (GAPDH) mRNA expression levels were measured using Power SYBR-Green Master Mix (Life Technologies, Carlsbad, CA, USA), and primers for the MTA family genes and GAPDH were designed using Primer3 software (Biology Workbench version 3.2; San Diego Super Computer Center, University of California, San Diego, CA, USA). The sequence information of these primers is provided in Table I. We performed 40 cycles of amplification under the following conditions: denaturation at $95^{\circ} \mathrm{C}$ for $10 \mathrm{sec}$, annealing at $60^{\circ} \mathrm{C}$ for $10 \mathrm{sec}$, and elongation at $72^{\circ} \mathrm{C}$ for $20 \mathrm{sec}$. After amplification, the products were subjected to a temperature gradient ranging from 68 to $95^{\circ} \mathrm{C}$ at $0.2^{\circ} \mathrm{C} / \mathrm{sec}$ under continuous fluorescence monitoring to produce a melting curve of the products. After proportional background adjustment, the fit-point method was used to determine the cycle in which the log-linear signal was distinguished from the background, and that cycle number was used as a crossing-point value. Expression levels of target transcripts and GAPDH were evaluated using Applied Biosystems StepOne software v2.1.

Relative mRNA expression analysis. The relative gene expression level determined by real-time quantitative RT-PCR was calculated using the standard curve method. Standard curves and linear equations were generated using 5 -fold serial dilutions of random-primed qPCR Human Reference cDNA (Takara Bio, Inc., Shiga, Japan; Clontech Laboratories, Mountain View, CA, USA). Within the range analyzed, all standard curves were linear with an acceptable correlation coefficient (R2). The extent of target mRNA expression was calculated from the standard curve, with quantitative normalization of the cDNA in each sample performed using the GAPDH gene as an internal control. Finally, target mRNA levels were expressed as ratios relative to the GAPDH mRNA level. Real-time PCR assays were performed in duplicate for each sample and the mean value was used to calculate mRNA expression levels.

Immunohistochemical analysis. Immunohistochemical studies of MTA1 were performed on surgical specimens of primary GC using avidin-biotin-peroxidase methods (DakoCytomation, Carpinteria, CA, USA) on formalin-fixed, paraffin-embedded (FFPE) tissues. The FFPE specimens were sliced into sections of 2-3 $\mu \mathrm{m}$. After deparaffinization and dehydration, specimens were brought to a boil in $10 \mathrm{mM}$ sodium citrate buffer for antigen unmasking. Specimens were then blocked and incubated with primary antibody overnight at $4^{\circ} \mathrm{C}$. The antibody was detected using Envision reagents (Envision kit/HRP; DakoCytomation, Glostrup, Denmark). A primary mouse polyclonal antibody against MTA1 (1:100; Santa Cruz Biotechnology, Inc., Santa Cruz, CA, USA) was used for implementing the labeled streptavidin-biotin method (LASB2 kit/HRP) and then stained with 3,3'-diaminobenzidine (both from DakoCytomation). All sections were counterstained with hematoxylin, and were dehydrated and mounted. Negative controls were also run simultaneously.

Statistical analysis. Results are expressed as the mean \pm SE, and all statistical analyses were performed using MedCalc version 12.3.0 (MedCalc Software, Mariakerke, Belgium). Differences between groups were estimated by the Mann-Whitney U test and the Kruskal-Wallis test, as applicable. For time-to-event analyses, survival estimates were calculated using Kaplan-Meier analysis and groups were compared with the log-rank test. Receiver operating characteristic (ROC) curves were established to determine the cut-off values for analyzing prognosis by Youden's index. Overall survival (OS) was measured from the date the patient underwent surgery until the date of death resulting from any cause, or last known follow-up for patients that were still alive. Disease-free survival (DFS) analysis was measured from the date the patient underwent curative surgery to the date of 
Table I. Primers used for quantitative real-time PCR analysis.

\begin{tabular}{llc}
\hline Gene & \multicolumn{1}{c}{ Forward } & Reverse \\
\hline MTA1 & CCTGCTGGCAGATAAAGGAG & GCTTGTCTGTGAGTGGGTTG \\
$M T A 2$ & ATGGAAATGTGGAGGCAAAG & GAAAAAGTTCCCGGTGCTTC \\
$M T A 3$ & AAAATGCCCACCCAGTCAG & TTTGGACTCCCAGTGTTTCG \\
GAPDH & GGAAGGTGAAGGTCGGAGTC & AATGAAGGGGTCATTGATGG
\end{tabular}

MTA, metastasis-associated protein; GAPDH, glyceraldehyde 3-phosphate dehydrogenase.
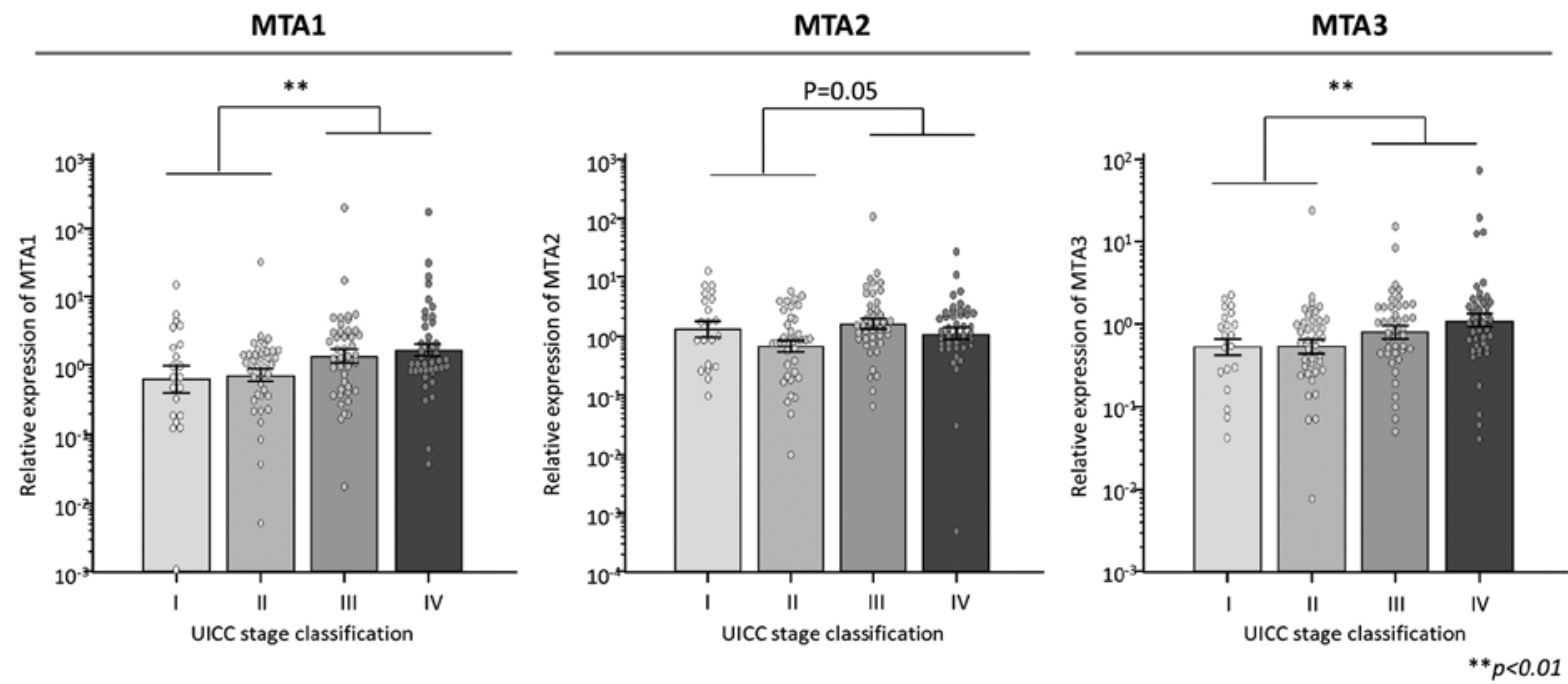

Figure 1. Expression status of metastasis-associated (MTA) protein family members in gastric cancer (GC) tissues according to TNM stage classification. Box plots showing the expression levels of all three MTA family members (MTA1, 2, and 3) in primary tumor tissues (GC) from 145 patients enrolled in this study. The expression of all MTA family members was correlated with disease progression (MTA1, $\mathrm{P}<0.01$; MTA2, $\mathrm{P}=0.05$; MTA3, $\mathrm{P}<0.01$ ). Boxes represent interquartile ranges, and the horizontal line across each box indicates the median mean \pm SE. The $y$-axis represents the relative expression of the three MTAs, and data were normalized to glyceraldehyde 3-phosphate dehydrogenase (GAPDH) expression. Statistical analysis was performed using Mann-Whitney $\mathrm{U}$ tests. All statistical tests were two-sided. ${ }^{* *} \mathrm{P}<0.01$

disease recurrence, death from any cause (i.e., cancer-unrelated deaths were not censored) or until last contact with the patient. For assessment of the performance as a prognostic marker for OS and DFS, the power calculations are based on the detection difference of 0.05 between favorable and unfavorable prognosis groups. We estimated 126 and 88 patients (distributed equally between the two groups) were needed to achieve $80 \%$ power to substantiate more than 25 and $30 \%$ differences in prognostic and recurrent outcomes at a significance level of 0.05 using a two-sided log-rank test, respectively, and our cohort of 145 GC patients was therefore more than adequate. The Cox's proportional hazards models were used to estimate hazard ratios (HRs) for death. Assumption of proportionality was confirmed for the Cox proportional hazards analyses by generating Kaplan-Meier survival curves (e.g., high vs. low expression groups) and by ensuring that the two curves did not intersect each other. Multivariate logistic regression models were used to predict factors influencing lymph node metastasis. Forced-entry regression was used to include these variables in all multivariable equations to analyze whether each of the predictors affected the outcome after adjusting for known confounders. All P-values were two-sided, and those $<0.05$ were considered statistically significant.

\section{Results}

Overexpression of MTA family proteins is associated with tumor progression in GC patients. Expression levels of MTA1, 2 and 3 in 145 GC tissues were examined by quantitative real-time PCR. The expression levels of both MTA1 and 3 were significantly higher in the advanced neoplastic tissues compared with these levels in early-stage GC tissues (MTA1, P<0.01; MTA2, P=0.05; MTA3, P<0.01; Fig. 1).

High expression of the MTA family proteins is associated with recurrence and poor outcome in GC patients. Next, we analyzed the expression patterns of the MTA family members with various clinicopathological factors to determine whether the expression status had any prognostic significance in GC patients (Table II). Elevated expression levels of all MTAs were significantly correlated with progression of TNM stage classification. Furthermore, overexpression of MTA1 and 3 was associated with factors showing metastasis such as presence of lymph node metastasis and distant metastasis in GC patients.

Moreover, in performing the time-to-event analysis to evaluate the prognostic impact of these MTAs, the expres- 
Table II. Clinicopathological variables and MTA family expression in GC patients.

\begin{tabular}{|c|c|c|c|c|c|c|c|}
\hline Variables & $\mathrm{n}$ & $\begin{array}{c}\text { MTA1 } \\
\text { expression }\end{array}$ & P-value & $\begin{array}{c}\text { MTA2 } \\
\text { expression }\end{array}$ & P-value & $\begin{array}{c}\text { MTA3 } \\
\text { expression }\end{array}$ & P-value \\
\hline \multicolumn{8}{|l|}{ Gender } \\
\hline Male & 116 & $5.59 \pm 2.22$ & \multirow[t]{2}{*}{0.66} & $3.07 \pm 0.94$ & \multirow[t]{2}{*}{0.67} & $2.21 \pm 0.69$ & \multirow[t]{2}{*}{0.46} \\
\hline Female & 29 & $2.09 \pm 0.53$ & & $2.31 \pm 0.46$ & & $1.28 \pm 0.28$ & \\
\hline \multicolumn{8}{|l|}{ Age (years) } \\
\hline$<70^{\mathrm{a}}$ & 71 & $2.67 \pm 0.57$ & \multirow[t]{2}{*}{0.94} & $2.27 \pm 0.41$ & \multirow[t]{2}{*}{0.23} & $1.38 \pm 0.32$ & \multirow[t]{2}{*}{0.7} \\
\hline$\geq 70$ & 74 & $7.02 \pm 3.44$ & & $3.54 \pm 1.44$ & & $2.65 \pm 1.04$ & \\
\hline \multicolumn{8}{|l|}{ Histological type } \\
\hline Intestinal type & 74 & $3.86 \pm 2.28$ & \multirow[t]{2}{*}{0.56} & $2.08 \pm 0.28$ & \multirow[t]{2}{*}{0.89} & $1.98 \pm 0.98$ & \multirow[t]{2}{*}{0.62} \\
\hline Diffuse type & 71 & $5.95 \pm 2.76$ & & $3.8 \pm 1.52$ & & $2.08 \pm 0.51$ & \\
\hline \multicolumn{8}{|l|}{ Tumor size } \\
\hline$\geq 5.5 \mathrm{~cm}^{\mathrm{b}}$ & 72 & $7.21 \pm 3.52$ & \multirow[t]{2}{*}{0.79} & $3.47 \pm 1.46$ & \multirow[t]{2}{*}{0.6} & $2.47 \pm 1.03$ & \multirow[t]{2}{*}{0.33} \\
\hline$<5.5 \mathrm{~cm}$ & 73 & $2.6 \pm 0.62$ & & $2.37 \pm 0.44$ & & $1.59 \pm 0.44$ & \\
\hline \multicolumn{8}{|c|}{ Pathological T category } \\
\hline $\mathrm{pT} 1 / 2$ & 49 & $1.57 \pm 0.33$ & \multirow[t]{2}{*}{0.15} & $2.04 \pm 0.35$ & \multirow[t]{2}{*}{0.42} & $0.87 \pm 0.09$ & \multirow[t]{2}{*}{0.25} \\
\hline pT3/4 & 96 & $6.58 \pm 2.67$ & & $3.37 \pm 1.13$ & & $2.62 \pm 0.83$ & \\
\hline \multicolumn{8}{|c|}{ Lymph node metastasis } \\
\hline N0 & 42 & $2.28 \pm 0.82$ & \multirow[t]{2}{*}{$0.009^{\mathrm{c}}$} & $2.01 \pm 0.4$ & \multirow[t]{2}{*}{0.27} & $1.35 \pm 0.55$ & \multirow[t]{2}{*}{0.07} \\
\hline N1 & 103 & $5.95 \pm 2.48$ & & $3.29 \pm 1.06$ & & $2.31 \pm 0.75$ & \\
\hline \multicolumn{8}{|c|}{ Distant metastasis } \\
\hline M0 & 102 & $3.9 \pm 1.9$ & \multirow[t]{2}{*}{0.08} & $3.16 \pm 1.04$ & \multirow[t]{2}{*}{0.78} & $1.3 \pm 0.28$ & \multirow[t]{2}{*}{$0.021^{\mathrm{c}}$} \\
\hline M1 & 43 & $7.24 \pm 3.98$ & & $2.35 \pm 0.65$ & & $3.75 \pm 1.74$ & \\
\hline \multicolumn{8}{|c|}{ TNM classification } \\
\hline Stage I/II & 60 & $1.89 \pm 0.57$ & \multirow[t]{2}{*}{$0.007^{\mathrm{c}}$} & $1.87 \pm 0.3$ & \multirow[t]{2}{*}{0.05} & $1.14 \pm 0.39$ & \multirow[t]{2}{*}{$0.007^{\mathrm{c}}$} \\
\hline Stage III/IV & 85 & $7.0 \pm 3.0$ & & $3.66 \pm 1.27$ & & $2.65 \pm 0.9$ & \\
\hline
\end{tabular}

${ }^{a}$ The median age at surgery was 70 years in this cohort, using the Mann-Whitney $\mathrm{U}$ test. ${ }^{\mathrm{b}}$ The median primary tumor size was $5.5 \mathrm{~cm}$ in this cohort. ${ }^{\mathrm{C}} \mathrm{P}<0.05$. MTA, metastasis-associated protein; GC, gastric cancer.

MTA1

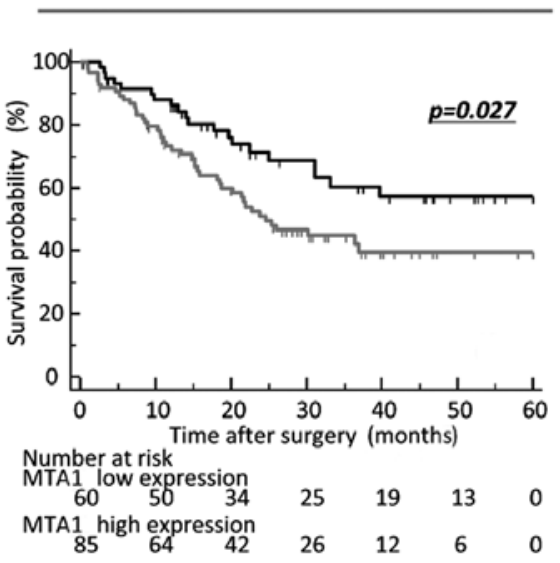

MTA2

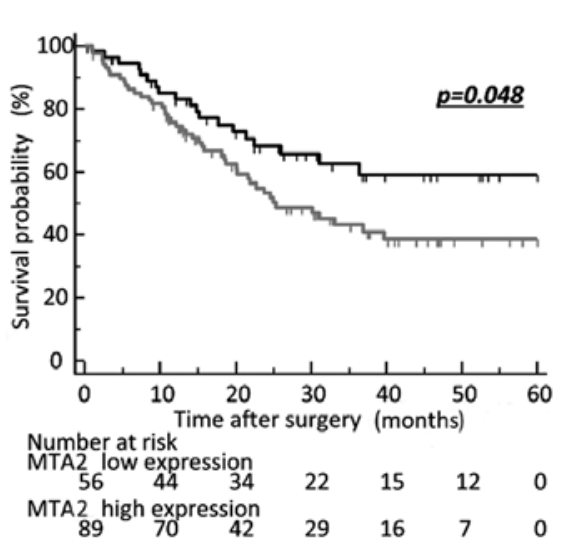

MTA3

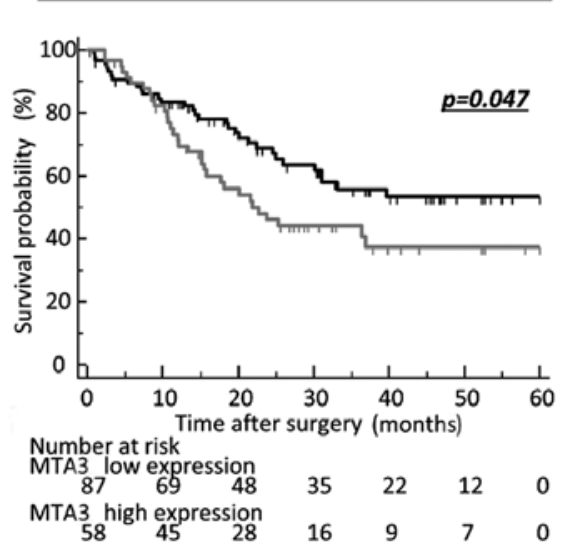

Figure 2. Prognostic impact of metastasis-associated (MTA) protein expression status in overall survival (OS) of gastric cancer (GC) patients. Kaplan-Meier survival curves for OS in GC patients based on the expression of three MTAs ( $\mathrm{n}=145)$. The OS rate in GC patients with high MTA expression in tumor tissue was significantly lower than the OS rate in GC patients with low MTA expression (MTA1, P=0.027; MTA2, P=0.048; MTA3, P=0.047; log-rank test). All statistical tests were two-sided.

sion cut-off threshold for each MTA family member was determined according to ROC analyses with Youden's index to analyze the prognostic impact of OS and DFS. Notably, high expression of all the MTA family was significantly 
MTA1

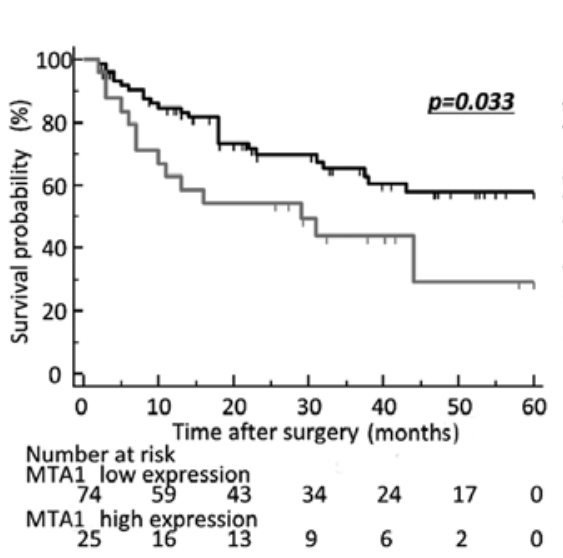

MTA2

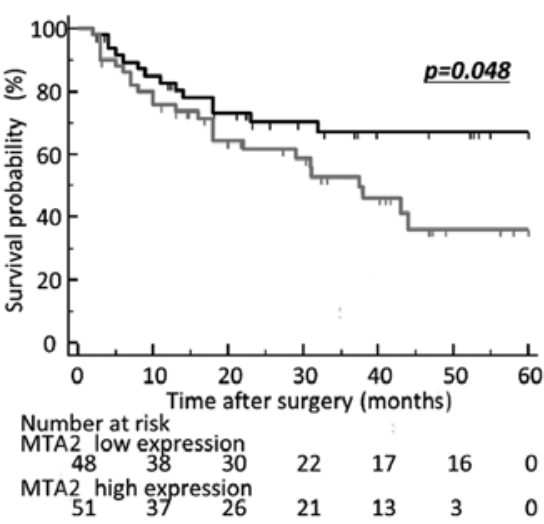

МTA3

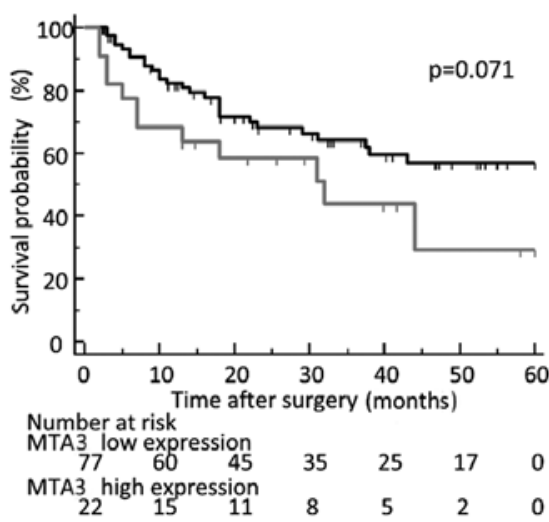

Figure 3. Prognostic impact of metastasis-associated (MTA) protein expression status in disease-free survival (DFS) of gastric cancer (GC) patients. DFS analyses based on the MTA expression status in GC tissue cohorts $(\mathrm{n}=99)$. Overexpression of both MTA1 and 2 were significantly correlated with poor DFS (MTA1, P=0.033; MTA2, P=0.048; MTA3, P=0.071; log-rank test). All statistical tests were two-sided.

Table III. Multivariate analysis for predictors of OS.

\begin{tabular}{|c|c|c|c|c|c|c|}
\hline \multirow[b]{2}{*}{ Variables } & \multicolumn{3}{|c|}{ Univariate } & \multicolumn{3}{|c|}{ Multivariate } \\
\hline & $\mathrm{HR}$ & $95 \% \mathrm{CI}$ & P-value & HR & $95 \% \mathrm{CI}$ & P-value \\
\hline Gender (male) & 0.79 & $0.44-1.43$ & 0.45 & 0.63 & $0.32-1.24$ & 0.18 \\
\hline Age $(\geq 70 \text { years })^{\mathrm{a}}$ & 1.16 & $0.71-1.89$ & 0.56 & 1.56 & $0.91-2.66$ & 0.11 \\
\hline Histological type (intestinal type) & 0.96 & $0.59-1.57$ & 0.86 & 1.19 & $0.7-1.99$ & 0.52 \\
\hline Tumor size $(\geq 5.5 \mathrm{~cm})^{\mathrm{b}}$ & 1.45 & $0.88-2.37$ & 0.14 & 1.61 & $0.95-2.73$ & 0.07 \\
\hline $\mathrm{T}$ classification (pT3/4) & 3.37 & $1.79-6.33$ & $0.0002^{\mathrm{d}}$ & 2.23 & $0.89-5.61$ & 0.09 \\
\hline Lymph node metastasis (present) & 3.57 & $1.77-7.22$ & $0.0004^{\mathrm{d}}$ & 1.92 & $0.71-5.19$ & 0.2 \\
\hline Distant metastasis (present) & 3.63 & $2.22-5.95$ & $<0.0001^{\mathrm{d}}$ & 2.79 & $1.52-5.1$ & $0.0009^{\mathrm{d}}$ \\
\hline $\begin{array}{l}\text { TNM stage classification } \\
\text { (Stage III/IV) }\end{array}$ & 5.03 & $2.67-9.47$ & $<0.0001^{\mathrm{d}}$ & 1.28 & $0.39-4.24$ & 0.69 \\
\hline High MTA1 expression ${ }^{c}$ & 1.8 & $1.06-3.05$ & $0.029^{\mathrm{d}}$ & 2.01 & $1.02-3.96$ & $0.044^{\mathrm{d}}$ \\
\hline High MTA2 expression ${ }^{c}$ & 1.71 & $1.00-2.92$ & 0.05 & 1.22 & $0.68-2.19$ & 0.51 \\
\hline High MTA3 expression $^{c}$ & 1.64 & $1.00-2.67$ & $0.049^{\mathrm{d}}$ & 0.88 & $0.47-1.62$ & 0.68 \\
\hline
\end{tabular}

${ }^{a}$ The median age at surgery was 70 years in GC patients. ${ }^{\mathrm{b}}$ The median primary size tumor was $5.5 \mathrm{~cm}$ in GC patients. ${ }^{\mathrm{c}} \mathrm{Cut}-\mathrm{off}$ threshold of expression for each MTA family member was determined by ROC analysis with Youden's index for overall survival in GC patients. ${ }^{\mathrm{d}} \mathrm{P}<0.05$. OS, overall survival; HR, hazard ratio; MTA, metastasis-associated protein; GC, gastric cancer.

correlated with poor prognosis compared with GCs in the low-expression group in terms of OS in these patients (MTA1, $\mathrm{P}=0.027$; MTA2, $\mathrm{P}=0.048$; MTA3, $\mathrm{P}=0.047$; log-rank test; Fig. 2). Furthermore, elevated expression of both MTA1 and 2 were significantly correlated with poor DFS (MTA1, $\mathrm{P}=0.033$; MTA2, $\mathrm{P}=0.048$; MTA3, $\mathrm{P}=0.071$; log-rank test; Fig. 3). Multivariate Cox's regression analysis showed that high MTA1 expression was an independent prognostic factor for OS (HR, 2.01; 95\% CI, 1.02-3.96; $\mathrm{P}=0.044)$ in GC patients (Table III).

High expression of MTAl is a predictive factor for the presence of lymph node metastasis in GC patients. Of the MTA family members, only MTA1 expression served as an independent prognostic factor for OS in GC patients. Furthermore, overexpression of MTA1 was significantly correlated with the presence of lymph node metastasis and recurrence in GC patients. Based on these findings, we next undertook multivariate logistic analysis to determine the clinical significance of MTA1 expression as a predictive biomarker of lymph node metastasis in GC patients (Table IV). Notably, advanced T classification and high expression of MTA1 were independent factors for lymph node metastasis. Collectively, our data suggest that tissue expression of MTA1 could be used as a predictive biomarker of lymph node metastasis and identification of high-risk GC patients.

MTAl was highly expressed in cancer cells compared with the cancer stroma or corresponding NM. To further confirm the pathological expression pattern of MTA1 in clinical specimens, 
Table IV. Multivariate analysis for lymph node metastasis.

\begin{tabular}{|c|c|c|c|c|c|c|}
\hline \multirow[b]{2}{*}{ Variables } & \multicolumn{3}{|c|}{ Univariate } & \multicolumn{3}{|c|}{ Mutivariate } \\
\hline & Odds ratio & $95 \% \mathrm{CI}$ & P-value & Odds ratio & $95 \% \mathrm{CI}$ & P-value \\
\hline Gender (male) & 1.38 & $0.58-3.29$ & 0.47 & 1.63 & $0.63-4.2$ & 0.32 \\
\hline Age $(\geq 70 \text { years })^{a}$ & 1.59 & $0.77-3.28$ & 0.21 & 2.23 & $0.98-5.06$ & 0.056 \\
\hline Histological type (Intestinal type) & 1.39 & $0.67-2.85$ & 0.37 & 1.55 & $0.69-3.48$ & 0.28 \\
\hline Tumor size $(\geq 5.5 \mathrm{~cm})^{\mathrm{b}}$ & 1.12 & $0.55-2.3$ & 0.75 & 1.18 & $0.52-2.7$ & 0.68 \\
\hline T classification (pT3/4) & 2.68 & $1.27-5.64$ & $0.009^{\mathrm{d}}$ & 3.19 & $1.38-7.34$ & $0.007^{\mathrm{d}}$ \\
\hline High MTA1 expression $^{c}$ & 2.48 & $1.19-5.17$ & $0.014^{\mathrm{d}}$ & 2.68 & $1.04-6.9$ & $0.041^{\mathrm{d}}$ \\
\hline High MTA2 expression $^{c}$ & 2.23 & $1.07-4.64$ & $0.031^{\mathrm{d}}$ & 1.87 & $0.81-4.31$ & 0.14 \\
\hline High MTA3 expression $^{c}$ & 1.73 & $0.81-3.71$ & 0.15 & 0.83 & $0.32-2.15$ & 0.7 \\
\hline
\end{tabular}

${ }^{a}$ The median age at surgery was 70 years in GC patients. ${ }^{b}$ The median primary tumor size was $5.5 \mathrm{~cm}$ in GC patients. ${ }^{\mathrm{c}} \mathrm{Cut-off}$ threshold expression level of each MTA family member was determined by ROC analysis with Youden's index for overall survival in GC patients. ${ }^{\mathrm{d}} \mathrm{P}<0.05$. MTA, metastasis-associated protein; GC, gastric cancer; ROC, receiver operating characteristic.

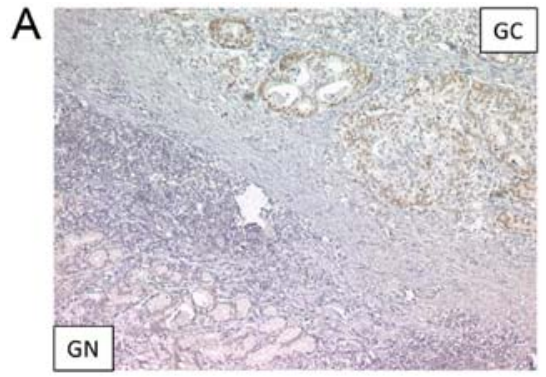

B
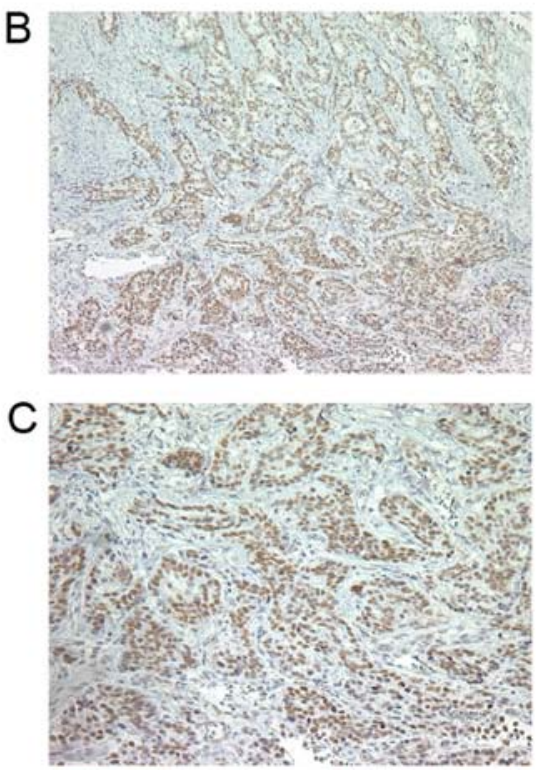

Figure 4. Immunohistochemical analysis of metastasis-associated protein 1 (MTA1) expression in gastric cancer (GC) tissues and corresponding normal mucosa (NM). MTA1 was overexpressed in the nuclei of primary GC cells but was not expressed (A) in NM or in the cancer stroma (B, magnification $\mathrm{x} 100$; C, magnification $\mathrm{x} 200$ ) in GC tissues.

immunohistochemical analysis was performed on 10 primary GC tissues and corresponding adjacent NM from this cohort. Immunohistochemical analysis revealed nuclear staining for MTA1 in GC cells, an observation that is consistent with previous reports in other types of cancer (17-19). Furthermore, the MTA1 protein was predominantly expressed in the nuclei of primary GC cells but exhibited no expression in NM and in the cancer stroma (Fig. 4A-C). Based on these results, MTA1 was overexpressed in the GC cells compared with the cancer stroma or NM, and its expression was significantly correlated with disease progression in GC patients.

\section{Discussion}

An increasing number of studies have implicated the involvement of the MTA family in disease progression and metastatic processes in various types of cancer (17,20-23), but the association between the expression pattern of MTA family members and their clinical significance in GC, particularly their potential as prognostic and predictive biomarkers for metastasis, have not yet been defined. In the present study, we provide initial evidence of the clinical impact of the dysregulation of the MTA family in GC. Firstly, expression of almost all of the MTA family members in primary tissues showed a significant correlation with the presence of metastasis and advanced TNM stage classification in GC patients. Secondly, high expression levels of all MTA family members were significantly correlated with poor survival, and in particular elevated expression of MTA1 in primary tissues was significantly correlated with poor prognosis for both OS and DFS. MTA1 expression was found to be an independent prognostic factor in GC patients. Thirdly, we showed that overexpression of MTA1 was deeply involved with lymph node metastasis and an independent risk factor for lymph node metastasis in GC.

The MTA tumor gene family was originally identified by differential cDNA screening of rat adenocarcinoma cell lines with low and high metastatic potential (9), and mainly consists of three different gene products, MTA1, 2 and 3 (7,8,24-26), which are critical components of the nucleosome remodeling and histone deacetylase (NuRD) complex that plays a negative role in posttranslational modifications of different genes by recruiting histone deacetylases onto their target genes (10). MTA1 was the first member of this family to be identified, and is a ubiquitously expressed protein that markedly 
increases metastasis and aggressiveness in various types of solid cancers, including esophageal carcinoma, thymoma, and ovarian, breast and colorectal cancer (27-32). A recent series of studies revealed a fundamental role for MTA1 in oncogenesis, DNA damage response, epithelial-mesenchymal transcription, inflammation, and pathogen-driven cancers by repressing transcription in a context-dependent manner, controlling the steady state of proteins via affecting protein ubiquitination, and contributing to the DNA damage response $(7,10,33)$. Two other members, MTA 2 and 3, were subsequently identified and found to be present in distinct NuRD complexes. MTA2 is a member of a highly-conserved family of proteins, and is structurally homologous to MTA1. MTA2 expression induces p53 deacetylation by HDACs and inhibits p53-dependent transcriptional activation. MTA3 was first identified as an estrogen-inducible gene product that forms a distinct Mi-2/NuRD complex (34), and recent evidence demonstrated that the depletion of MTA3 expression inhibited cell proliferation and induced cell cycle arrest at the G1/S boundary in non-small lung cancer cell lines due to suppression of cyclin A, cyclin D1 and pRb (23). Similar to MTA1, MTA2 and 3 dysregulation has also been demonstrated in several tumors, including pancreatic, ovarian, and non-small cell lung cancer, and gastroesophageal junction adenocarcinoma $(23,35-39)$.

One of the major findings of the present study is the prognostic impact of the MTA family expression levels in GC patients. The expression status of all MTA family members was significantly correlated with metastasis factors and advanced TNM stage in GC patients. In addition, elevated expression of all of MTA family members showed significant correlation with poor prognosis in OS. Our results are consistent with previous studies in various types of cancer. Recently, several studies used a series of in vitro and in vivo experiments to demonstrate that the MTA family members contribute to GC cell proliferation, migration and invasion via dysregulation of downstream target genes, including cyclin D1, interleukin-11, fibronectin, matrix metalloproteinase (MMP)-2 and -9 (40,41). Collectively, these studies together with our data suggest the intimate involvement of the MTA family members in disease progression and formation of metastasis in GC.

Another key finding of our study was the clinical impact of MTA1 expression in GC patients. Our results clearly demonstrated that high expression of MTA1 was a potential predictor for recurrence and poor prognosis in GC patients. In line with these findings, multivariate analysis revealed that high expression of MTA1 was an independent risk factor for survival in GC patients. Notably, high expression of MTA1 was significantly correlated with the presence of lymph node metastasis, and logistic regression analysis showed that elevated MTA1 expression in primary cancer tissues was an independent risk factor for predicting lymph node metastasis in GC patients. At present, one of the most clinically desired biomarkers for GC patients is a predictive biomarker for identifying patients with lymph node metastasis. Lymph node metastasis is recognized as one of the most important risk factors for recurrence and prognosis in GC patients, and accumulating evidence has revealed that lymph node dissection improves survival (42). Furthermore, thanks to recent advances in endoscopic and surgical techniques over the past decade, accurate detection of lymph node metastasis has become more clinically relevant by converting to minimally invasive treatments, such as endoscopic resection or laparoscopic-assisted gastrectomy for patients with early GC. Therefore, identification of GC patients with lymph node metastasis using molecular biomarkers such as MTA1 expression will help the surgeon in decision-making regarding lymph node dissection for these patients during surgery, and allow the endoscopist to select subgroups of high-risk early-stage GC patients with lymph node metastasis.

In conclusion, this study provides novel evidence for the clinical impact of the expression of the MTA family in GC patients. Our systemic assessment highlights the clinical feasibility of MTA family members, in particular MTA1, as prognostic and predictive biomarkers for lymph node metastasis and recurrence in GC patients. We conclude that assessment of MTA1 expression in primary tumors may be used as a clinically relevant prognostic and predictive biomarker in GC patients. Quantification of MTA1 expression may support the accurate diagnosis of disease staging and may help predict clinical outcomes.

\section{Acknowledgements}

The authors would like to thank Motoko Ueeda and Yuki Orito for their excellent technical assistance. Dr Yoshinaga Okugawa was supported in part by a grant in Aid for Scientific Research from Takeda Science Foundation, Japan. The present study was also supported in part by a grant in Aid for Scientific Research (no. 25462018) from the Ministry of Education, Culture, Sports, Science and Technology, Japan (to M.O.).

\section{References}

1. Siegel R, Naishadham D and Jemal A: Cancer statistics, 2013. CA Cancer J Clin 63: 11-30, 2013.

2. Gupta GP and Massagué J: Cancer metastasis: building a framework. Cell 127: 679-695, 2006.

3. ten Kate M, Hofland LJ, van Grevenstein WM, van Koetsveld PV, Jeekel $\mathbf{J}$ and van Eijck $\mathrm{CH}$ : Influence of proinflammatory cytokines on the adhesion of human colon carcinoma cells to lung microvascular endothelium. Int J Cancer 112: 943-950, 2004.

4. Basoglu M, Yildirgan MI, Taysi S, Yilmaz I, Kiziltunc A, Balik AA, Celebi F and Atamanalp SS: Levels of soluble intercellular adhesion molecule-1 and total sialic acid in serum of patients with colorectal cancer. J Surg Oncol 83: 180-184, 2003.

5. Fidler IJ: Critical determinants of metastasis. Semin Cancer Biol 12: 89-96, 2002.

6. Chan DA and Giaccia AJ: Hypoxia, gene expression, and metastasis. Cancer Metastasis Rev 26: 333-339, 2007.

7. Toh Y and Nicolson GL: The role of the MTA family and their encoded proteins in human cancers: molecular functions and clinical implications. Clin Exp Metastasis 26: 215-227, 2009.

8. Kumar R, Wang RA, Mazumdar A, Talukder AH, Mandal M, Yang Z, Bagheri-Yarmand R, Sahin A, Hortobagyi G, Adam L, et al: A naturally occurring MTA1 variant sequesters oestrogen receptor-alpha in the cytoplasm. Nature 418: 654-657, 2002.

9. Toh Y, Pencil SD and Nicolson GL: A novel candidate metastasis-associated gene, mta1, differentially expressed in highly metastatic mammary adenocarcinoma cell lines. cDNA cloning, expression, and protein analyses. J Biol Chem 269: 22958-22963, 1994.

10. Li DQ, Pakala SB, Nair SS, Eswaran J and Kumar R: Metastasis-associated protein $1 /$ nucleosome remodeling and histone deacetylase complex in cancer. Cancer Res 72: 387-394, 2012.

11. Okugawa Y, Toiyama Y, Hur K, Toden S, Saigusa S, Tanaka K, Inoue Y, Mohri Y, Kusunoki M, Boland CR, et al: Metastasis-associated long non-coding RNA drives gastric cancer development and promotes peritoneal metastasis. Carcinogenesis 35: 2731-2739, 2014. 
12. Okugawa Y, Tanaka K, Inoue Y, Kawamura M, Kawamoto A, Hiro J, Saigusa S, Toiyama Y, Ohi M, Uchida K, et al: Brain-derived neurotrophic factor/tropomyosin-related kinase B pathway in gastric cancer. Br J Cancer 108: 121-130, 2013.

13. Okugawa Y, Inoue Y, Tanaka K, Kawamura M, Saigusa S, Toiyama Y, Ohi M, Uchida K, Mohri Y and Kusunoki M: Smad interacting protein 1 (SIP1) is associated with peritoneal carcinomatosis in intestinal type gastric cancer. Clin Exp Metastasis 30: 417-429, 2013.

14. Okugawa Y, Toiyama Y, Tanaka K, Matsusita K, Fujikawa H, Saigusa S, Ohi M, Inoue Y, Mohri Y, Uchida K, et al: Clinical significance of zinc finger E-box binding homeobox 1 (ZEB1) in human gastric cancer. J Surg Oncol 106: 280-285, 2012.

15. Toiyama Y, Tanaka K, Kitajima T, Shimura T, Imaoka H, Mori K, Okigami M, Yasuda H, Okugawa Y, Saigusa S, et al: Serum angiopoietin-like protein 2 as a potential biomarker for diagnosis, early recurrence and prognosis in gastric cancer patients. Carcinogenesis 36: 1474-1483, 2015.

16. Japanese Gastric Cancer Association. Japanese Classification of Gastric Carcinoma, 3rd English ed., 14: 101-112, 2011.

17. Dias SJ, Zhou X, Ivanovic M, Gailey MP, Dhar S, Zhang L, He Z, Penman AD, Vijayakumar S and Levenson AS: Nuclear MTA1 overexpression is associated with aggressive prostate cancer, recurrence and metastasis in African Americans. Sci Rep 3: 2331,2013

18. Pakala SB, Rayala SK, Wang RA, Ohshiro K, Mudvari P, Reddy SD, Zheng Y, Pires R, Casimiro S, Pillai MR, et al: MTA1 promotes STAT3 transcription and pulmonary metastasis in breast cancer. Cancer Res 73: 3761-3770, 2013.

19. Li WF, Liu N, Cui RX, He QM, Chen M, Jiang N, Sun Y, Zeng J, Liu LZ and Ma J: Nuclear overexpression of metastasis-associated protein 1 correlates significantly with poor survival in nasopharyngeal carcinoma. J Transl Med 10: 78, 2012.

20. Wang W, Yang ZL, Liu JQ, Yang LP, Yang XJ and Fu X Overexpression of MTA1 and loss of KAI-1 and KiSS-1 expressions are associated with invasion, metastasis, and poor-prognosis of gallbladder adenocarcinoma. Tumori 100: 667-674, 2014.

21. Covington KR, Brusco L, Barone I, Tsimelzon A, Selever J, Corona-Rodriguez A, Brown P, Kumar R, Hilsenbeck SG and Fuqua SA: Metastasis tumor-associated protein 2 enhances metastatic behavior and is associated with poor outcomes in estrogen receptor-negative breast cancer. Breast Cancer Res Treat 141: 375-384, 2013.

22. Liu T, Yang M, Yang S, Ge T, Gu L and Lou G: Metastasis-associated protein 1 is a novel marker predicting survival and lymph nodes metastasis in cervical cancer. Hum Pathol 44: 2275-2281, 2013.

23. Li H, Sun L, Xu Y, Li Z, Luo W, Tang Z, Qiu X and Wang E: Overexpression of MTA3 correlates with tumor progression in non-small cell lung cancer. PLoS One 8: e66679, 2013.

24. Manavathi B and Kumar R: Metastasis tumor antigens, an emerging family of multifaceted master coregulators. J Biol Chem 282: 1529-1533, 2007.

25. Manavathi B, Singh K and Kumar R: MTA family of coregulators in nuclear receptor biology and pathology. Nucl Recept Signal 5: e010, 2007.

26. Kumar R, Wang RA and Bagheri-Yarmand R: Emerging roles of MTA family members in human cancers. Semin Oncol 30 (Suppl 16): 30-37, 2003.
27. Qian H, Lu N, Xue L, Liang X, Zhang X, Fu M, Xie Y, Zhan Q, Liu Z and Lin C: Reduced MTA1 expression by RNAi inhibits in vitro invasion and migration of esophageal squamous cell carcinoma cell line. Clin Exp Metastasis 22: 653-662, 2005.

28. Sasaki H, Yukiue H, Kobayashi Y, Nakashima Y, Kaji M, Fukai I, Kiriyama M, Yamakawa Y and Fujii Y: Expression of the MTA1 mRNA in thymoma patients. Cancer Lett 174: 159-163, 2001.

29. Dannenmann C, Shabani N, Friese K, Jeschke U, Mylonas I and Brüning A: The metastasis-associated gene MTA1 is upregulated in advanced ovarian cancer, represses ERbeta, and enhances expression of oncogenic cytokine GRO. Cancer Biol Ther 7: 1460-1467, 2008.

30. Tong D, Heinze G, Schremmer M, Schuster E, Czerwenka K, Leodolter S and Zeillinger R: Expression of the human MTA1 gene in breast cell lines and in breast cancer tissues. Oncol Res 16: 465-470, 2007.

31. Tuncay Cagatay S, Cimen I, Savas B and Banerjee S: MTA-1 expression is associated with metastasis and epithelial to mesenchymal transition in colorectal cancer cells. Tumour Biol 34: 1189-1204, 2013.

32. Li J, Ye L, Sun PH, Satherley L, Hargest R, Zhang Z and Jiang WG: MTA1 is up-regulated in colorectal cancer and is inversely correlated with lymphatic metastasis. Cancer Genomics Proteomics 12: 339-345, 2015.

33. Avtanski DB, Nagalingam A, Kuppusamy P, Bonner MY, Arbiser JL, Saxena NK and Sharma D: Honokiol abrogates leptin-induced tumor progression by inhibiting Wnt1-MTA1- $\beta$-catenin signaling axis in a microRNA-34a dependent manner. Oncotarget 6: 16396-16410, 2015.

34. Fujita N, Jaye DL, Kajita M, Geigerman C, Moreno CS and Wade PA: MTA3, a Mi-2/NuRD complex subunit, regulates an invasive growth pathway in breast cancer. Cell 113: 207-219, 2003.

35. Ji Y, Zhang P, Lu Y and Ma D: Expression of MTA2 gene in ovarian epithelial cancer and its clinical implication. J Huazhong Univ Sci Technolog Med Sci 26: 359-362, 2006.

36. Chen DW, Fan YF, Li J and Jiang XX: MTA2 expression is a novel prognostic marker for pancreatic ductal adenocarcinoma. Tumour Biol 34: 1553-1557, 2013.

37. Liu SL, Han Y, Zhang Y, Xie CY, Wang EH, Miao Y, Li HY, $\mathrm{Xu}$ HT and Dai SD: Expression of metastasis-associated protein 2 (MTA2) might predict proliferation in non-small cell lung cancer. Target Oncol 7: 135-143, 2012.

38. Dong H, Guo H, Xie L, Wang G, Zhong X, Khoury T, Tan D and Zhang H: The metastasis-associated gene MTA3, a component of the Mi-2/NuRD transcriptional repression complex, predicts prognosis of gastroesophageal junction adenocarcinoma. PLoS One 8: e62986, 2013.

39. Zhou C, Ji J, Cai Q, Shi M, Chen X, Yu Y, Liu B, Zhu Z and Zhang J: MTA2 promotes gastric cancer cells invasion and is transcriptionally regulated by Sp1. Mol Cancer 12: 102, 2013.

40. Yao Y, Feng S, Xiao M, Li Y, Yang L and Gong J: MTA1 promotes proliferation and invasion in human gastric cancer cells. Onco Targets Ther 8: 1785-1794, 2015.

41. Zhou C, Ji J, Cai Q, Shi M, Chen X, Yu Y, Zhu Z and Zhang J: MTA2 enhances colony formation and tumor growth of gastric cancer cells through IL-11. BMC Cancer 15: 343, 2015.

42. Wu CW, Hsiung CA, Lo SS, Hsieh MC, Chen JH, Li AF, Lui WY and Whang-Peng J: Nodal dissection for patients with gastric cancer: a randomised controlled trial. Lancet Oncol 7: 309-315, 2006. 